\title{
Cost-effectiveness of once-daily vs twice-daily tacrolimus among Hispanic and Black kidney transplant recipients
}

\author{
Jason T Hurwitz, MS, PhD; Amy J Grizzle, PharmD; Carmelina S Tyler, PharmD, BCPS; \\ Lorenzo Villa Zapata, PharmD, PhD; and Daniel C Malone, PhD
}

\section{What is already known about this subject}

- Tacrolimus is a first-line immunosuppressive therapy to prevent rejection and graft failure in kidney transplant recipients, but some patient subpopulations are rapid metabolizers and require higher doses to achieve target trough levels.

- Once-daily extended-release tacrolimus tablets (LCPT) have a unique pharmacokinetic profile, which results in a lower peak and less peak-to-trough fluctuation compared with immediate-release tacrolimus taken twice daily (IR-Tac).

\section{What this study adds \\ - Use of LCPT in Hispanic and Black kidney transplant recipients during the first year of treatment is more effective and costs more than IR-Tac, indicating a trade-off scenario. \\ - The incremental cost-effectiveness ratio (ICER) for using LCPT during the first year of treatment over IR-Tac in the Hispanic group was $\$ 65,643$ per additional successfully treated patient who avoids treatment-related serious adverse events and graft loss with consequent dialysis; the ICER in the Black group was $\$ 90,458$.}

\section{Author affiliations \\ Jason T Hurwitz, MS, PhD, and Amy J Grizzle, PharmD, Center for Health Outcomes and PharmacoEconomic Research (HOPE Center), University of Arizona, Tucson. Carmelina S Tyler, PharmD, BCPS, Veloxis Pharmaceuticals, Inc., Cary, NC. Lorenzo Villa Zapata, PharmD, PhD, Department of Pharmacy Practice, College of Pharmacy, Mercer University, Atlanta, GA. Daniel C Malone, PhD, Department of Pharmacotherapy, College of Pharmacy, University of Utah, Salt Lake City.}

AUTHOR CORRESPONDENCE: Jason T Hurwitz, 520.626.8889; Hurwitz@pharmacy.arizona.edu

J Manag Care Spec Pharm 2021;27(7):948-60

Copyright $(2021$, Academy of Managed Care Pharmacy. All rights reserved.

1 year and without treatment-related SAEs. Probabilistic sensitivity analyses established distributions for cost and outcomes estimates, and a series of one-way sensitivity analyses identified parameters that had the most effect on results.

RESULTS: Total overall cost for the Hispanic group was $\$ 14,765$ for LCPT and $\$ 12,416$ for IR-Tac, and total cost in the Black group was $\$ 16,626$ for LCPT and $\$ 9,871$ for IR-Tac. Total overall effectiveness of LCPT and IR-Tac was $88.32 \%$ and $84.75 \%$ in the Hispanic group and $93.24 \%$ and $85.78 \%$ in the Black group, respectively. The incremental 
cost-effectiveness ratio (ICER) for using LCPT over IR-Tac during the first year of treatment in the Hispanic group was $\$ 65,643$ per additional successfully treated patient. The ICER for the Black group was $\$ 90,458$. The single parameter having the most impact on results in both groups was the probability of a treatment-related SAE in IR-Tac, which accounted for $49 \%$ of variation in results in the Hispanic group and $46 \%$ in the Black group.

CONCLUSIONS: Overall results for both groups show that LCPT is incrementally more costly and more effective compared with IR-Tac, indicating a trade-off scenario. LCPT is a cost-effective strategy if a decision makers' willingness to pay for 1 additional successfully treated patient exceeds the ICER and must be weighed against the costs of graft loss, continuing dialysis, and potential retransplant. This study provides a foundation for further research to update and expand inputs as more data become available to improve real-world relevance and decision making.

The primary goal of immunosuppressive therapy after kidney transplantation is to prevent rejection and graft failure. Although acute rejection is treatable, allowing the graft to continue functioning, graft failure is an irreversible loss that requires patients to return to dialysis and await another transplant. Tacrolimus, a calcineurin inhibitor, is currently approved for the prophylaxis of organ rejection in patients receiving allogeneic liver, kidney, or heart transplants. Tacrolimus is one of the most widely used immunosuppression drugs, and clinical guidelines recommend it as a first-line immunosuppressive therapy following kidney transplantation. ${ }^{1}$ However, tacrolimus has a narrow therapeutic index, and it is important to achieve therapeutic concentrations soon after transplant to avoid potentially toxic effects that result from higher concentrations or graft loss from underdosing. ${ }^{2}$

Since release of the current clinical guidelines by the Kidney Disease Improving Global Outcomes in 2009, advances in sequencing technologies and a growing body of genome-wide association studies have identified genotypes affecting CYP3A enzymes associated with pharmacokinetic differences in tacrolimus-centered immunosuppression that should be considered when prescribing. ${ }^{2}$ Black patients are much more likely than Caucasian patients to express the CYP3A5*1 wild-type allele associated with increased tacrolimus clearance and variability (ie, rapid metabolizers), ${ }^{2-6}$ and they may also experience low drug levels immediately following transplantation, delaying therapeutic tacrolimus levels. ${ }^{7}$ Because of this enzyme, Black patients often need higher doses to reach therapeutic levels, which leads to higher peak concentrations of tacrolimus. ${ }^{8}$ Some toxicities are associated with peak concentrations of tacrolimus. ${ }^{9}$ Similarly, compared with Caucasian patients, Hispanic patients may also be more likely to express the CYP3A5*1 wild-type allele and less likely to express the CYP3A5*3 allele associated with slower tacrolimus clearance. ${ }^{4,6,10-12}$ Prevalence of the CYP3A5*1 expression is also higher in East Asian populations-specifically Chinese, Japanese, and Korean-than in European populations..$^{3,5}$

Orally administered tacrolimus is available as an immediate-release capsule taken twice daily (IR-Tac; Prograf, Astellas Pharma US, Inc.); an extended-release capsule taken once daily (Astagraf XL, Astellas Pharma US, Inc., and Advagraf abroad); and an extended-release tablet taken once daily (LCPT; Envarsus XR, Veloxis Pharmaceuticals, Inc.). LCPT employs a MeltDose drug delivery technology that uses small particle size to alter pharmacokinetic properties, resulting in increased bioavailability and broader absorption in the gastrointestinal tract with a lower peak and less peak-to-trough fluctuation compared with IR-Tac. ${ }^{13-16}$ Due to these characteristics, LCPT requires on average a $20 \%$ lower dose than IR-Tac. ${ }^{17,18}$ In addition, reduced dosing from twice to once daily can also improve adherence, a major risk factor for graft failure..$^{19-21}$

Two phase 3 clinical trials demonstrated noninferior efficacy and similar safety between LCPT and IR-Tac overall. ${ }^{17,18}$ The first study was an open label trial of stable adult patients who received a kidney transplant 3 months to 5 years before enrollment and randomized to either continue with IR-Tac or convert to a reduced dose of LCPT. ${ }^{18}$ The second study was a double-blind, double-dummy trial of adult de novo patients randomized to receive LCPT or IR-Tac. ${ }^{17}$ These 2 studies together evaluated 861 patients (LCPT, N=428; IR-Tac, N=433) over a 1-year period, followed by further post hoc pooled and subgroup analyses. ${ }^{22-25}$

At 12 months, the overall incidence of treatment failure was statistically similar for LCPT and IR-Tac groups, defined as a composite endpoint including biopsy-proven acute rejection (BPAR), graft loss, death, or lost to follow-up. ${ }^{24}$ Subgroup analyses, however, showed that Black patients in the LCPT group experienced numerically fewer treatment failures and adverse events (AEs) than Black patients in the IR-Tac group (14\% less treatment failures overall, including $10 \%$ less BPAR and 2\% less graft loss, as well as $7 \%$ less with $>1$ treatment-emergent AE and 9\% less with $>1$ serious AE), ${ }^{24}$ whereas Hispanic de novo patients in the LCPT group experienced significantly less treatment failures (13\% less, $\mathrm{P}=0.04){ }^{23}$

Despite the perceived advantages of LCPT compared with IR-Tac, especially among rapid metabolizers, no studies have examined its cost-effectiveness. Recent data 


\section{FIGURE 1 Decision Tree Model (Same Structure for Hispanic and Black Groups)}

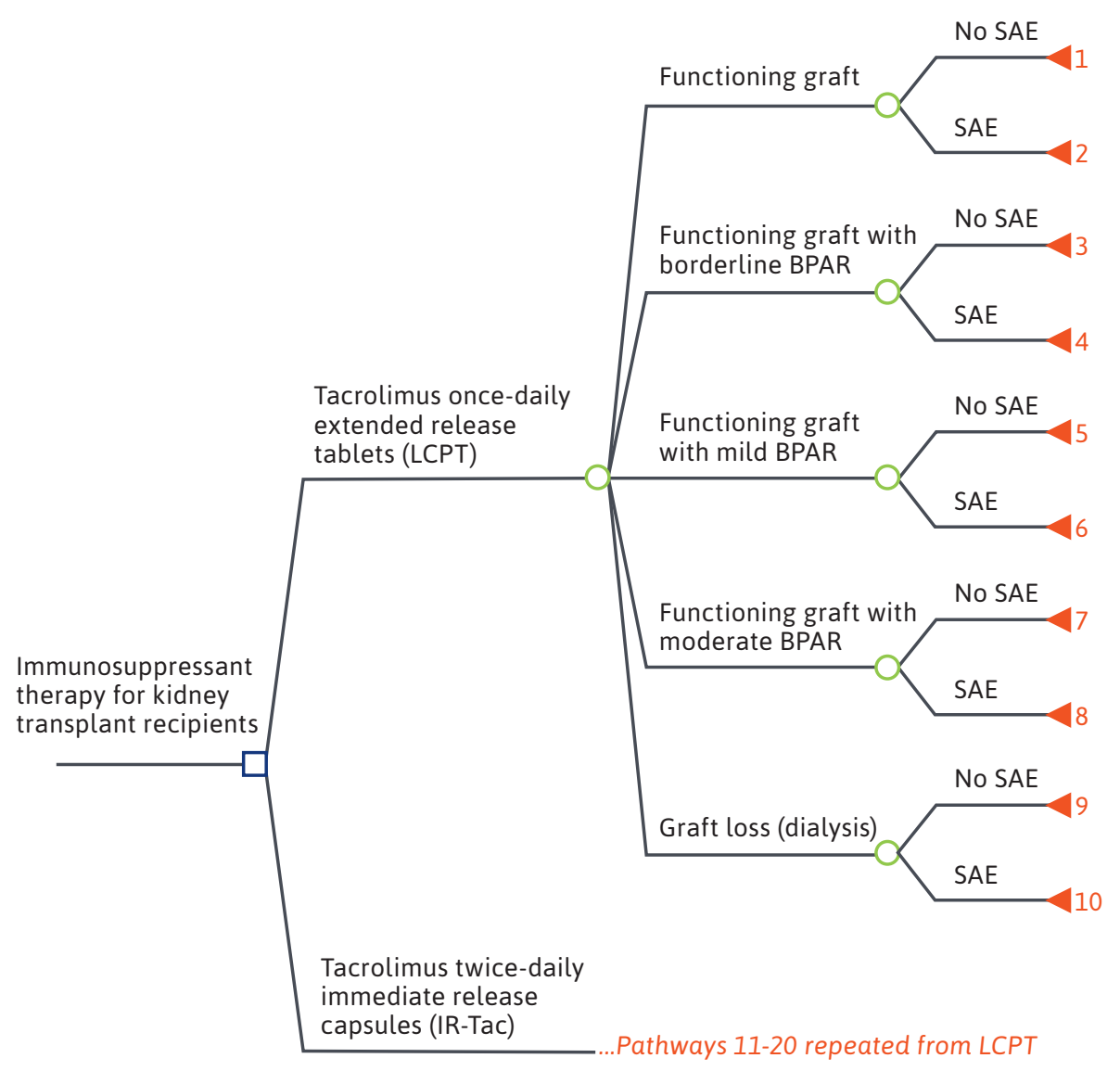

BPAR= biopsy proven acute rejection; $S A E=$ serious adverse event (treatment related).

available from the clinical trials provide an opportunity to pursue this topic. Specifically, the purpose of this study was to evaluate the cost-effectiveness of LCPT vs IR-Tac in Hispanic and Black kidney transplant recipient subpopulations.

\section{Methods}

\section{DESIGN}

Following approval by the University of Arizona Institutional Review Board, we developed a decision analytical model to evaluate the cost-effectiveness for the first year of LCPT vs IR-Tac therapy based on outcomes data for Hispanic and Black subgroups from the pooled clinical trials.17,18,22-24 Although the clinical trials were conducted in the United States and abroad, the treatment patterns in this model reflected current US practices, and the analysis perspective of this study was that of a US health care payer, such as a managed care organization. Similar outcomes data for Hispanic and Black patients from the other once-daily tacrolimus formulation were not publicly available at the time of this study to include in an indirect comparison.

\section{MODEL STRUCTURE AND OUTCOMES}

The decision tree model simulated the following possible outcomes, comprising 20 total pathways (Figure 1): (1) functioning graft, (2) functioning graft with borderline BPAR, (3) functioning graft with mild BPAR, (4) functioning graft with moderate BPAR, (5) graft loss and consequent dialysis, and (6) serious adverse event (SAE) related to the study drug.

Treatment-related SAEs may occur with any of the first 5 outcomes, resulting in 10 pathways each for LCPT and IR-Tac. Severe BPAR events, treatment-related deaths, and kidney retransplants did not occur in the selected patient populations during the 1-year clinical trial period and thus were excluded from the model. The model structure for the Hispanic and Black groups was identical and was analyzed separately using Microsoft Excel (2015) and decision tree modeling software (TreeAge Pro 2019, TreeAge Software, LLC).

The primary outcome in this model was a successfully treated patient, defined as having a functioning graft without treatment-related SAEs, regardless of BPAR (LCPT pathways 1, 3, 5, 7 and IR-Tac pathways 11, 13, 15, 17). In other words, treatment failure involved treatment-related SAEs or graft loss (returning to dialysis). This definition more closely reflects safety and efficacy endpoints in clinical practice, differing from the original clinical trials, which included BPAR as a treatment failure. . $^{17,18,22-24}$ Although BPAR was an unwanted complication, it was a treatable condition that resulted in a functioning kidney, which was the desired model outcome.

The primary result of this analysis was to calculate and interpret the incremental cost-effectiveness ratio (ICER), which indicated the cost per additional successfully treated patient using LCPT vs IR-Tac. The ICER value 
equaled the difference in total costs between LCPT and IR-Tac divided by the difference in probabilities of treatment success.

\section{RESOURCE USE}

Immunosuppressants. To capture variations in dosing regimens (eg, adjustments made to maintain trough concentrations of 6-11 ng/mL for the first 30 days, then $4-11 \mathrm{ng} / \mathrm{mL}$ for the remainder of the study), average doses were used from 4 dosing periods (days 1-30, 31-90, 91-180, and 181-360) for each patient group and treatment arm (clinical trial data on file). All model pathways included 1 year of immunosuppressant therapy (LCPT or IR-Tac) except for the graft loss pathway, which included only a partial year of immunosuppressant therapy based on the average time to graft loss (clinical trial data on file).

BPAR Treatments. The clinical trials did not collect information about treating borderline, mild, and moderate BPAR. Resource use associated with these outcomes therefore relied on the literature and author experience. ${ }^{26-34}$

To treat borderline BPAR, the model assumed a 1-day hospitalization that included a percutaneous renal biopsy and augmented dose of immunosuppressant. In addition, $50 \%$ of cases received intravenous methylprednisolone (500 mg daily for 3 days) through an outpatient infusion clinic, while also taking oral prednisone (40 $\mathrm{mg}$ taper over 5 days).

Mild and moderate BPAR treatments involved 3- and 5-day hospitalizations, respectively; percutaneous renal biopsy; augmented dose of immunosuppressant; intravenous methylprednisolone; and thymoglobulin. In addition, all mild BPAR cases received a $40 \mathrm{mg}$ oral prednisone taper over 5 days, whereas half the cases also resumed opportunistic infection prophylaxis comprising valganciclovir (450 mg daily for 90 days), sulfamethoxazole-trimethoprim (1 single-strength tablet daily for 90 days), and nystatin (20 mL daily for 30 days). In contrast, all moderate BPAR cases resumed the same opportunistic infection prophylaxis and received a more intensive prednisone regimen (60 mg taper over 40 days).

Graft Loss and Dialysis. Using the average number of days to graft loss by patient group and treatment arm (clinical trial data on file), the graft loss and dialysis pathway accounted for a partial year of immunosuppressant medication and hospitalization (average length of stay $=6.33$ days, standard error $[\mathrm{SE}]=0.25$, derived from HCUPnet using International Classification of Diseases, Tenth Revision, Clinical Modification [ICD-10-CM] diagnostic code T86.12 Kidney transplant failure) followed by dialysis for the remainder of the year. ${ }^{35}$
Treatment-Related SAEs. This analysis included AEs that resulted in hospitalizations, classified as "serious" by the US Food and Drug Administration, and reported by the clinical trial investigators as related to the study drugs (clinical trial data on file). ${ }^{36}$

\section{EVENT PROBABILITIES}

Movement through the decision tree was based on data extracted from the pooled analysis for Black patients ${ }^{17,18,24}$; 2 research abstracts focused on Hispanics from the pooled data $^{22,23}$; and additional data for each patient group (clinical trial data on file). The pooled clinical studies included specific information on the number and types of treatmentrelated SAEs, number and severity of BPAR events, and other information needed to estimate costs of resource use described elsewhere in this article (ie, average doses, proportions of 2019 US branded and generic tacrolimus prescriptions, and days to graft loss).

Many patients experienced multiple types of events during the 1-year study period. The number of patients who experienced 1 or more treatment-related SAEs determines the probability of occurrence, whereas the total numbers and types of SAEs determine the costs. Similarly, some subjects experienced multiple BPAR events with different severity levels during the study period. The probability of BPAR occurrence included anyone with 1 or more BPAR events categorized by their highest level of severity. For example, a patient who experienced 2 borderline and 1 mild BPAR events would appear in "mild BPAR." However, the per person cost incorporated the multiple BPARs experienced by a single person, calculated as the sum of costs associated with all events divided by the number of patients who experienced the events. Those who experienced BPAR and graft loss appear separately in the BPAR probability and costs, as well as in the graft loss probability and costs.

\section{DIRECT MEDICAL COSTS AND SOURCES}

The model included the following direct medical costs: LCPT and IR-Tac treatments; borderline, mild, and moderate BPAR; graft failure and resulting dialysis; and treatmentrelated SAEs. The costs for all outpatient medications in this analysis were based on current wholesale acquisition costs (WAC) retrieved on January 6, 2020. ${ }^{37}$ The WAC was \$5.31 per mg for LCPT, \$6.03 per mg for branded IR-Tac, and on average $\$ 1.54(\mathrm{SD}=\$ 0.96 ; \mathrm{n}=29)$ for generic IR-Tac. Generics included any active status drugs with tacrolimus active ingredient administered orally as capsules, excluding repackagers. Further, IR-Tac costs reflected the proportion of generic and branded 2019 US prescription claims by patient subgroup, with generic comprising $94.42 \%$ and 92.06\% of the claims among Hispanic and Black patients 
nationwide. ${ }^{38}$ Thus, the final IR-Tac costs used in this study were \$1.79 per mg and \$1.90 per mg in the Hispanic and Black groups.

Costs to treat BPAR derived from multiple sources. The hospitalization costs associated with all 3 levels of BPAR were based on 2016 national weighted estimates from HCUPnet National Inpatient Sample using the ICD-10-CM diagnostic code (T86.11 Kidney transplant rejection) and adjusted for a daily average (ie, average total cost divided by average length of stay). ${ }^{35}$ HCUPnet is a database of acute care encounters across the United States and is broadly representative of inpatient services and costs. Hospitalization costs were assumed to include a percutaneous biopsy, augmented dose of immunosuppressant, and, for mild and moderate BPAR, intravenous methylprednisolone and thymoglobulin. If augmented dosing of LCPT or IR-Tac continued after discharge, then it was assumed already captured within the average daily dosage (clinical trial data on file). Costs of oral medications taken as an outpatient were based on WAC pricing. ${ }^{37}$ For borderline BPAR, the cost of administering intravenous methylprednisolone at an outpatient infusion clinic was based on current Medicare Physician Fee Schedule for CPT \#96365 "Intravenous infusion, hydration; initial, 31 minutes to 1 hour." 39,40

Hospitalization costs related to graft loss also derived from HCUPnet using ICD-10-CM diagnostic code T86.12 Kidney transplant failure. ${ }^{35}$ In addition, this pathway included the costs of a partial year of medication (LCPT or IR-Tac) until the average time to graft loss (for a given treatment arm), followed by dialysis costs for the remainder of the year. The average number of days on dialysis for Hispanic patients was $237.75(\mathrm{n}=2, \mathrm{SE}=290.75)$ in LCPT and $241.75(n=4, S E=282.98)$ in IR-Tac (clinical trial data on file). No Black patients experienced graft loss in the LCPT arm, while only 1 patient experienced it in the IR-Tac arm $(176.25$ days). ${ }^{24}$ Dialysis costs were from the recent US Renal Data System 2019 Annual Report. ${ }^{41}$ The clinical trials did not collect information on the type of dialysis patients received, so the model assumed the proportions of hemodialysis and peritoneal dialysis recipients in the United States by patient subgroups. ${ }^{41}$

We linked the list of treatment-related SAEs (clinical trial data on file) with ICD-10-CM codes (Supplementary Table 1, available in online article) to identify hospitalization cost estimates. ${ }^{35}$ To calculate per-person costs, we summed the hospitalization costs for all treatment-related SAEs and divided by the number of patients who experienced at least 1 event.

The hospitalization costs associated with BPAR, graft loss, and treatment-related SAEs were adjusted for inflation to 2019 US dollars using the Consumer Price Index "hospital services" series, and dialysis costs were adjusted using the "medical care" series. ${ }^{42}$

\section{ASSUMPTIONS}

The model components, structure, and interpretation of results rested on several assumptions. First, the model assumed that all patients start with a functioning graft. Hence, the model did not include delayed graft function, where newly transplanted kidneys require temporary dialysis until the kidney "wakes up" nor any pretransplant tacrolimus costs or resources used. The model also assumed that generic and branded twice-daily immediaterelease tacrolimus are clinically equivalent and have the same dosing and that all patients self-administered LCPT tablets and IR-Tac capsules. Although BPAR may be associated with increased risk of graft loss, ${ }^{43}$ without patient-level data the model assumed that graft loss risk did not differ between those with and without BPAR history and that model parameters (events) were uncorrelated. Concomitant immunosuppressant use was also assumed to be equivalent between treatment groups.

Subgroup level data were unavailable, but the most frequently used concomitant immunosuppressants overall in the first clinical trial by treatment group (LCPT [n=162]/IR-Tac [ $\mathrm{n}=162])$ included mycophenolate mofetil $(60 \% / 66 \%)$, mycophenolic acid $(34 \% / 31 \%)$, and corticosteroids $(66 \% / 74 \%)$ (clinical trial data on file) ${ }^{18}$ whereas all patients in the second clinical trial received corticosteroids per local use (requiring at least $5 \mathrm{mg}$ of prednisone for 12 months), mycophenolate mofetil (2 g/day), and an IL-2 receptor anatagonist induction with basiliximab (clinical trial data on file). ${ }^{17}$

The analysis included 3 subjects lost to follow-up in the IR-Tac arm for 52 days on average $(\mathrm{SD}=26)$ after starting treatment. During this time, 1 subject experienced a graft loss and was included in the "graft loss/dialysis" branch of the model, so the subsequent loss to follow-up was nonconsequential. However, the other 2 subjects experienced neither graft loss nor BPAR during their study participation and so appear in the "functioning graft" branch. This favors IR-Tac because the potential for BPAR or graft loss events for these subjects would otherwise continue in that arm for the remaining approximately 10 months.

\section{SENSITIVITY ANALYSES}

The model used a probabilistic sensitivity analysis, based on 5,000 Monte Carlo simulations, to account for uncertainty or variability in all inputs. Cost ranges were based on variability estimates previously mentioned using a gamma distribution, while uncertainty in effects were based on 


\section{TABLE 1 Demographic Characteristics of Study Sample}

\begin{tabular}{|c|c|c|c|c|c|c|c|c|}
\hline \multirow{3}{*}{$\begin{array}{ll} & \text { Parameter } \\
\text { Age, mean years (SD) } & \end{array}$} & \multicolumn{4}{|c|}{ Hispanic } & \multicolumn{4}{|c|}{ Black } \\
\hline & \multicolumn{2}{|c|}{ LCPT $(n=100)$} & \multicolumn{2}{|c|}{ IR-Tac $(n=108)$} & \multicolumn{2}{|c|}{ LPCT $(n=44)$} & \multicolumn{2}{|c|}{ IR-Tac $(n=49)$} \\
\hline & 39.4 & $(13.7)$ & 40.6 & $(15.1)$ & 50.1 & $(10.3)$ & 47.2 & $(12.8)$ \\
\hline \multicolumn{9}{|l|}{ Age group, $n(\%)$} \\
\hline$<65$ years & 95 & $(95.0)$ & 100 & $(92.6)$ & 39 & $(88.6)$ & 44 & $(89.8)$ \\
\hline$\geq 65$ years & 5 & $(5.0)$ & 8 & $(7.4)$ & 5 & (11.4) & 5 & (10.2) \\
\hline \multicolumn{9}{|l|}{ Sex, n (\%) } \\
\hline Female & 30 & $(30.0)$ & 30 & $(27.8)$ & 13 & $(29.5)$ & 18 & $(36.7)$ \\
\hline Male & 70 & $(70.0)$ & 78 & $(72.2)$ & 31 & $(70.5)$ & 31 & $(63.3)$ \\
\hline \multicolumn{9}{|l|}{ Race, $n(\%)$} \\
\hline White & 62 & $(62.0)$ & 66 & $(61.1)$ & 0 & $(0.0)$ & 0 & $(0.0)$ \\
\hline Black/African American & 0 & $(0.0)$ & 3 & $(2.8)$ & 44 & $(100.0)$ & 49 & $(100.0)$ \\
\hline Asian & 0 & $(0.0)$ & 2 & (1.9) & 0 & $(0.0)$ & 0 & $(0.0)$ \\
\hline American Indian/Alaska Native & 0 & $(0.0)$ & 1 & $(0.9)$ & 0 & $(0.0)$ & 0 & $(0.0)$ \\
\hline Other & 38 & $(38.0)$ & 36 & $(33.3)$ & 0 & $(0.0)$ & 0 & $(0.0)$ \\
\hline \multicolumn{9}{|l|}{ Donor type, $n$ (\%) } \\
\hline Deceased & 34 & $(34.0)$ & 40 & 37.0) & 26 & $(59.1)$ & 35 & $(71.4)$ \\
\hline Living & 66 & $(66.0)$ & 68 & $(63.0)$ & 18 & $(40.9)$ & 14 & $(28.6)$ \\
\hline \multicolumn{9}{|l|}{ Transplant status, n (\%) } \\
\hline De novo & 74 & $(74.0)$ & 79 & $(73.1)$ & 9 & $(20.5)$ & 15 & $(30.6)$ \\
\hline Stable & 26 & $(26.0)$ & 29 & $(26.9)$ & 35 & $(79.5)$ & 34 & $(69.4)$ \\
\hline \multicolumn{9}{|l|}{ Diabetes at time of transplant, $n(\%)$} \\
\hline Yes & 29 & $(29.0)$ & 23 & $(21.3)$ & 17 & $(38.6)$ & 18 & $(36.7)$ \\
\hline No & 71 & $(71.0)$ & 85 & $(78.7)$ & 27 & $(61.4)$ & 31 & $(63.3)$ \\
\hline Days from transplant to first dose of treatment, mean (SD) & 190.2 & $(417.4)$ & 146.5 & $(313.5)$ & 631.5 & $(548.3)$ & 427.6 & $(457.8)$ \\
\hline Days on treatment, mean (SD) & 352.3 & $(100.9)$ & 336.5 & $(124.0)$ & 326.4 & $(102.9)$ & 344.5 & $(89.6)$ \\
\hline Baseline weight in $\mathrm{kg}$, mean (SD) & 72.2 & $(16.6)$ & 74.3 & (18.9) & 87.5 & $(15.2)$ & 86.9 & $(20.2)$ \\
\hline BMI $\left(\mathrm{kg} / \mathrm{m}^{2}\right)$, mean (SD)a & 25.9 & $(5.2)$ & 26.4 & $(5.5)$ & 30.2 & $(5.8)$ & 29.8 & $(7.2)$ \\
\hline$<30 \mathrm{~kg} / \mathrm{m}^{2}, \mathrm{n}(\%)^{\mathrm{a}}$ & 78 & $(78.0)$ & 79 & $(73.1)$ & 24 & $(54.5)$ & 24 & $(49.0)$ \\
\hline$\geq 30 \mathrm{~kg} / \mathrm{m}^{2}, \mathrm{n}(\%)^{\mathrm{a}}$ & 22 & $(22.0)$ & 29 & (26.9) & 17 & $(38.6)$ & 22 & $(44.9)$ \\
\hline
\end{tabular}

Source: Clinical trial data on file.

Note: There were no statistically significant differences between treatment arms within each group (ie, $P>0.05)$.

${ }^{a}$ BMI data in the Black group were available for only 41 LCPT and 46 IR-Tac patients.

$B M I=$ body mass index; $L C P T=$ tacrolimus once-daily extended-release tablets; IR-Tac=tacrolimus twice-daily immediate-release capsules

proportions observed in the clinical trial using a beta distribution.

A follow-up series of one-way analyses used the lower and upper ranges of all parameters to examine the independent impact of each parameter on overall results. These deterministic analyses used the same cost ranges previously noted but vary estimates $\pm 20 \%$ for each probability instead of using a beta distribution. For example, 13.64\% of the 44 Black patients in the LCPT arm experienced borderline BPAR, with a $\pm 20 \%$ range of $10.91 \%-16.37 \%$ (clinical trial data on file). For events with no patients, such as graft loss/dialysis in this same group, 1 was added and the upper value became $2.27 \%(1 / 44)$, while the lower value remained at $0 \%(0 / 44) .{ }^{24}$ Upper values for high frequency events, such as $84.09 \%(37 / 44)$ of this group that experienced functioning graft without BPAR, capped at 100\% (clinical trial data 


\section{TABLE 2 Per Patient Resource Use, Clinical Event Costs, and Event Probabilities During the First Year of Treatment}

\begin{tabular}{|c|c|c|c|c|c|}
\hline \multirow[b]{2}{*}{ Hispanic, n } & \multicolumn{2}{|c|}{ LCPT } & \multicolumn{2}{|c|}{ IR-Tac } & \multirow{2}{*}{$\begin{array}{c}\text { Source } \\
22,23^{a}\end{array}$} \\
\hline & 100 & & 108 & & \\
\hline \multicolumn{6}{|l|}{ Health states, n (\%) ${ }^{\mathrm{b}}$} \\
\hline \multicolumn{6}{|l|}{ Branch $1^{c}$} \\
\hline Functioning graft & 83 & $(83.0)$ & 82 & $(75.9)$ & $22,23^{a}$ \\
\hline Functioning graft: borderline BPAR & 7 & $(7.0)$ & 6 & $(5.6)$ & a \\
\hline Functioning graft: mild BPAR & 8 & $(8.0)$ & 8 & (7.4) & a \\
\hline Functioning graft: moderate BPAR & 0 & $(0.0)$ & 8 & $(7.4)$ & a \\
\hline Graft loss (dialysis) & 2 & $(2.0)$ & 4 & $(3.7)$ & 22,23 \\
\hline \multicolumn{6}{|l|}{ Branch 2} \\
\hline$>1$ treatment-related SAE & 10 & $(10.0)$ & 13 & $(12.0)$ & a \\
\hline \multicolumn{6}{|l|}{ First-year costs, mean (SE) ${ }^{d}$} \\
\hline Medication ${ }^{e}$ & 9,319 & $(624)$ & 3,663 & $(235)$ & $37,38^{a}$ \\
\hline Treatment-related $\mathrm{SAE}^{\mathrm{f}}$ & 24,521 & $(1,935)$ & 21,599 & $(1,489)$ & $35^{\mathrm{a}}$ \\
\hline Borderline BPAR & 4,281 & $(158)$ & 4,281 & $(158)$ & $35,37,39,40^{a}$ \\
\hline Mild BPAR & 13,903 & $(817)$ & 13,903 & $(817)$ & $35,37^{a}$ \\
\hline Moderate BPAR & 23,701 & $(1,487)$ & 23,701 & $(1,487)$ & $35,37^{a}$ \\
\hline Graft lossg & 90,475 & $(20,604)$ & 89,471 & $(22,428)$ & $35,37,38,41^{a}$ \\
\hline Tacrolimus & 3,253 & $(218)$ & 1,239 & $(80)$ & $37,38^{a}$ \\
\hline Hospitalization & 27,200 & $(1,578)$ & 27,201 & $(1,578)$ & 35 \\
\hline Dialysis & 60,022 & $(18,808)$ & 61,032 & $(20,770)$ & $41^{\mathrm{a}}$ \\
\hline
\end{tabular}

on file). A separate series of deterministic and one-way sensitivity analyses were also used for periodic diagnostic and validity checks during model construction.

\section{Results}

Internal clinical trial analyses showed no statistically significant differences in sample characteristics between treatment arms within the Hispanic and Black patient groups (Table 1; clinical trial data on file). Among the Hispanic (LCPT, $\mathrm{n}=100$; IR-Tac, $\mathrm{n}=108$ ) and Black (LCPT, $\mathrm{n}=44$; IR-Tac, $\mathrm{n}=49$ ) patient groups, 3 patients self-identified as both Hispanic and Black, appearing in each of the separate analyses. This study excluded White non-Hispanic patients who also participated in the clinical trials. Table 2 summarizes the probabilities of clinical events, the average per-patient clinical event costs, and overall resource use in the first 12 months used to populate the decision tree model. Unit level costs per resource use and clinical event are provided in Supplementary Table 2 (available in online article).

Total overall average costs for the Hispanic group were $\$ 14,765$ in the LCPT arm and $\$ 12,416$ in the IR-Tac arm, whereas total costs for the Black group were $\$ 16,626$ for LCPT and $\$ 9,871$ for IR-Tac (Supplementary Table 3 , available in online article). These cost values represent the "fully-loaded" cost estimates over 1 year, accounting for complications (ie, BPAR and treatment-related SAEs) and graft-loss/dialysis. Total effectiveness represents the probability of a successfully treated patient without treatment-related SAEs and graft loss/dialysis. The total effectiveness in the Hispanic group was $88.32 \%$ in the LCPT arm and $84.75 \%$ in the IR-Tac arm, while the total effectiveness in the Black group was $93.24 \%$ for LCPT and $85.78 \%$ in IR-Tac.

Results for Hispanic and Black groups show that use of LCPT was more effective and cost more than using IR-Tac. In the Hispanic group, the average ICER for using LCPT instead of IR-Tac was $\$ 65,643$ per additional successfully 


\section{TABLE 2}

Per Patient Resource Use, Clinical Event Costs, and Event Probabilities During the First Year of Treatment (continued)

\begin{tabular}{|c|c|c|c|c|c|}
\hline \multirow[b]{2}{*}{ Black, n } & \multicolumn{2}{|c|}{ LCPT } & \multicolumn{2}{|c|}{ IR-Tac } & \multirow{2}{*}{$\begin{array}{c}\text { Source } \\
24\end{array}$} \\
\hline & 44 & & 49 & & \\
\hline \multicolumn{6}{|l|}{ Health states, n (\%) ${ }^{\mathrm{b}}$} \\
\hline \multicolumn{6}{|l|}{ Branch $1^{c}$} \\
\hline Functioning graft & 37 & $(84.1)$ & 40 & $(81.6)$ & $24^{a}$ \\
\hline Functioning graft: borderline BPAR & 6 & $(13.6)$ & 2 & $(4.1)$ & a \\
\hline Functioning graft: mild BPAR & 1 & $(2.3)$ & 5 & $(10.2)$ & a \\
\hline Functioning graft: moderate BPAR & 0 & $(0.0)$ & 1 & $(2.0)$ & a \\
\hline Graft loss (dialysis) & 0 & $(0.0)$ & 1 & (2.0) & 24 \\
\hline \multicolumn{6}{|l|}{ Branch 2} \\
\hline >1 Treatment-related SAE & 3 & $(6.8)$ & 6 & $(12.2)$ & a \\
\hline \multicolumn{6}{|l|}{ First-year costs, mean (SE) ${ }^{d}$} \\
\hline Medicatione & 15,038 & $(1,122)$ & 4,927 & (382) & $37,38^{a}$ \\
\hline Treatment-related SAE & 10,132 & $(416)$ & 11,737 & (144) & $35^{\mathrm{a}}$ \\
\hline Borderline BPAR & 4,281 & $(158)$ & 4,281 & (158) & a \\
\hline Mild BPAR & 13,903 & $(817)$ & 13,903 & $(817)$ & a \\
\hline Moderate BPAR & 23,701 & $(1,487)$ & 23,701 & $(1,487)$ & a \\
\hline Graft lossg & - & - & 75,990 & $(1,776)$ & $35,37,38,41^{a}$ \\
\hline Tacrolimus & - & - & 2,550 & (198) & $37,38^{a}$ \\
\hline Hospitalization & - & - & 27,200 & $(1,578)$ & 35 \\
\hline Dialysis & - & - & 46,240 & - & $41^{\mathrm{a}}$ \\
\hline
\end{tabular}

${ }^{a}$ Clinical trial data on file.

${ }^{b}$ Number of study subjects who experienced events within 12 months of starting treatment. Beta distributions were used for all probabilities, where $\alpha=e v e n t s$ out of $\beta=$ total sample.

'Although some patients may have experienced multiple levels of BPAR over time (borderline, mild, or moderate), the numbers reported here reflect only the highest level observed per patient.

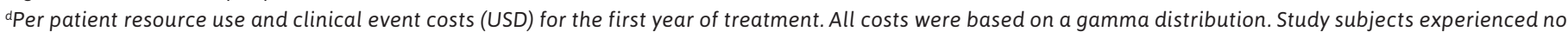
"severe" BPAR events so are not included here.

${ }^{e}$ Adjusted for the average daily dosing of study subjects and for IR-Tac, the market share proportion for twice-daily tacrolimus between branded (Prograf) and generic. The branded product accounted for 5.58\% and 7.94\% of all 2019 twice-daily tacrolimus prescription claims among Hispanic and Black populations (respectively), while the generic comprised the remainder.

'Adjusted for inflation to 2019 USD using the CPI for hospital services.

${ }^{9}$ Based on (1) 2016 costs for ICD-10-CM diagnostic code T86.12 "Kidney transplant failure," plus (2) the costs for the average number of days on dialysis per group, and (3) costs of medication for the balance of the year. Dialysis costs were adjusted for the proportion of hemodialysis and peritoneal dialysis recipients in the United States in 2017. Dialysis costs were adjusted for inflation to 2019 USD using CPI medical care values, while the cost of "kidney transplant failure" was adjusted using CPI hospital services values.

$B P A R=$ biopsy proven acute rejection; $C P I=$ Consumer Price Index; ICD-10-CM = International Classification of Diseases, Tenth Revision, Clinical Modification; $I R-T a C=$ tacrolimus twice-daily immediate-release capsules; $L C P T=$ tacrolimus once-daily extended-release tablets; $S A E=$ serious adverse event; SE =standard error; USD $=$ United States dollars.

treated patient who avoided treatment-related SAEs and graft loss with consequent dialysis during the first year of treatment. In the Black group, the ICER was $\$ 90,458$.

In addition, the cost-effectiveness acceptability curve for the 2 immunosuppressant therapies in Figure 2 displays the probabilities of either being cost-effective over a range of willingness-to-pay (WTP) values. This is particularly useful when a decision maker's WTP threshold value is unknown. For example, at a WTP threshold of $\$ 150,000$ per additional successfully treated patient, LCPT was cost-effective in $64 \%$ of the simulations for the Hispanic group and in $67 \%$ for the Black group. 


\section{FIGURE 2 Cost-Effectiveness Acceptability Curve for Hispanic and Black Subgroups}

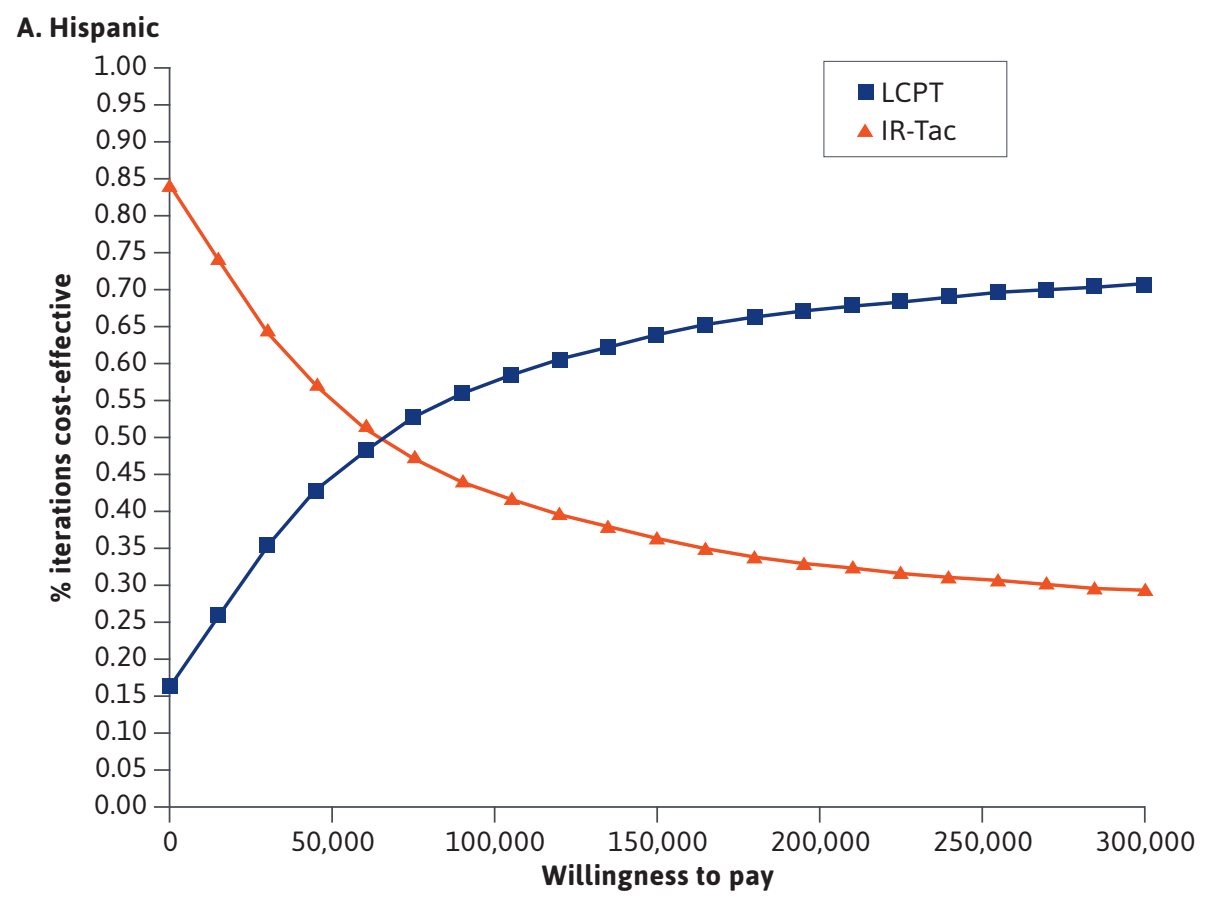

B. Black

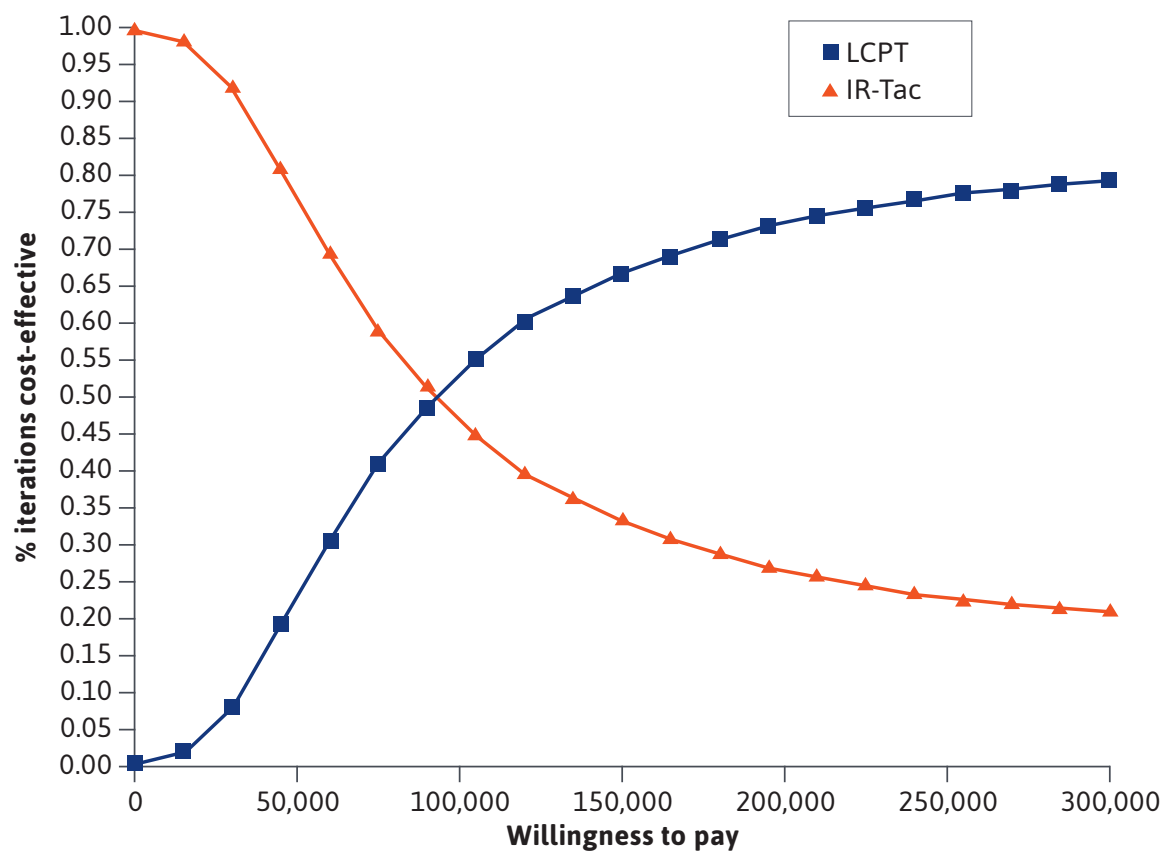

Note: The curved lines intersect at the WTP equal to the ICERs for each group. As WTP increases, so does the probability that LCPT will be a cost-effective strategy.

$I C E R=$ incremental cost-effectiveness ratio; IR-TaC = tacrolimus twice-daily immediate-release capsule; $L C P T=$ tacrolimus once-daily extended-release tablets; WTP = willingness to pay.
A series of one-way sensitivity analyses identified parameters that independently had the greatest influence on the ICERs within each group. Of the 66 model parameters included, 11 in the Hispanic group and 7 in the Black group accounted for over 99\% of significant changes in ICER values for each group, while the remainder of the 66 parameters in each group had little to no impact (Supplementary Figure 1, available in online article). Six parameters common to driving the results in the Hispanic and Black groups were the medication costs for LCPT and IR-Tac, the probabilities of graft loss for both medications, and the probabilities of treatment-related SAEs for both medications.

The parameter with the most impact in both groups was the probability of a treatment-related SAE in IR-Tac, which accounted for $49 \%$ of variation in results in the Hispanic group and $46 \%$ in the Black group. Substituting the base-case value for this parameter with the low range value (ie, a lower probability of treatment-related SAE in IR-Tac) favored IR-Tac and would have increased the original ICERs from $\$ 65,643$ to $\$ 243,106$ in the Hispanic group and from $\$ 90,458$ to $\$ 145,963$ in the Black group. Conversely, using a high range value favored LCPT and would have decreased the original ICERs to a lesser extent from $\$ 65,643$ to $\$ 31,286$ in the Hispanic group and from $\$ 90,458$ to $\$ 67,096$ in the Black group. For other parameters, such as the cost of LCPT or the probability of treatment-related SAE in LCPT, high values favored IR-Tac and would have increased ICERs, whereas low values favored LCPT and would have decreased ICERs.

\section{Discussion}

In the Hispanic and Black subpopulations, LCPT was incrementally more costly and more effective compared 
with IR-Tac. The ICERs for the Black $(\$ 90,458)$ and Hispanic $(\$ 65,643)$ groups represent the cost of using LCPT during the first year of treatment over IR-Tac to achieve 1 additional successfully treated patient who avoided treatment-related SAEs and graft loss with consequent dialysis. For these subpopulations, LCPT may be useful because of its specific pharmacogenomic characteristics affecting pharmacokinetics of tacrolimus. ${ }^{2-6,10-12}$

LCPT appears to be a cost-effective strategy, given the relatively low ICER values. In general, when a decision maker's WTP for 1 additional successfully treated patient exceeds the ICER, the technology should be reimbursed and made available. While no known accepted WTP thresholds exist for these outcomes, one may weigh these ICERs against the costs of treating graft loss, resuming dialysis for an indefinite period of months or years, and potential retransplant. Adjusted for inflation to 2019 US dollars, Medicare spent $\$ 96,253$ per patient per year for hemodialysis and $\$ 81,954$ for peritoneal dialysis ${ }^{41}$ while the median cost for a deceased donor transplant is $\$ 125,033$, including the cost of organ acquisition and hospital care. ${ }^{44}$ Patient quality of life and the potential for additional complications must also be considered. Even 1 year of dialysis followed by retransplant exceeds the additional cost of using LCPT.

Costs of LCPT and IR-Tac medications were among the several parameters that most influenced results in the Hispanic and Black analyses. These were based on WAC, but payers may substitute with their actual costs. Current tacrolimus costs are especially difficult to estimate, given the price fluctuations due to continued global shortages reported since May 2019.45,46 During the time that we incorporated these inputs, the average cost per milligram of branded IR-Tac remained the same, while generic IR-Tac, the largest proportion of the overall IR-Tac cost, increased by $10.4 \%$ and LCPT increased by $2.5 \%{ }^{37}$ The effect on results of an additional WAC increase of IR-Tac over LCPT would only favor LCPT further. If we updated our model using these current costs, the ICERs for LCPT would be even more favorable.

Similar outcomes data for Hispanic and Black patients are not publicly available elsewhere to include as indirect comparisons at this time. As more data become available, researchers can update and expand inputs from this model to improve real-world decision making. Additional research is needed, for example, to compare the long-term costs and effectiveness beyond the first year of treatment, incorporate other outcomes such as the quality-of-life impacts of preventing graft loss and dialysis, and evaluate additional comparators such as another once-daily tacrolimus formulation currently available.

\section{LIMITATIONS}

A unique feature of this study is the direct comparison between LCPT and IR-Tac based on actual, rather than simulated, event probabilities from clinical trial data for the 2 groups. However, presence of so few or zero events (eg, no moderate BPAR and graph loss/dialysis events among Blacks in the LCPT arm) lacks variation for probabilistic sensitivity analyses. Given such small numbers, a single extra graft loss in either arm can dramatically affect results. These results are based on randomized clinical trial data and may not be generalizable to real-world settings where patient outcomes may be less favorable.

Some data that may improve parameter estimates, such as the actual health care resource utilization of the clinical trial patients following BPAR and treatment-related SAEs, were also unavailable. Although generalizability to real-world settings is an inherent limitation of randomized clinical trials, all costs included in this model were based on real-world sources.

The one-way sensitivity analyses explored a series of "what if" scenarios associated with the extreme ranges of values of all parameters, which may be realistic but rare occurrences. Given the single source of outcomes data, this analysis assigned $\pm 20 \%$ to all probability values to gauge potential effects on ICER results. However, future research can assign different \pm percentage ranges or update these ranges as more data sources become available.

\section{Conclusions}

Overall results for both groups show that LCPT is incrementally more costly and more effective compared with IR-Tac, indicating a trade-off scenario. The ICERs for Hispanic $(\$ 65,643)$ and Black $(\$ 90,458)$ groups represent the cost of using LCPT over IR-Tac to achieve 1 additional successfully treated patient who avoids treatment-related SAEs and graft loss with consequent dialysis. LCPT is a costeffective strategy because of the relatively low ICER value, especially if weighed against the costs of graft loss, continuing dialysis, and potential retransplant. As more data become available and tacrolimus prices stabilize following the shortage, researchers can build on this model to update and expand inputs to improve real-world relevance and decision making. 


\section{DISCLOSURES}

This study was funded by Veloxis Pharmaceuticals, Inc., which provided clinical trial file data and nonbinding feedback on the model structure, data interpretation, clinical expertise, manuscript review, and areas of publication interest (ie, managed care). Hurwitz, Grizzle, Villa Zapata, and Malone received grant funding from Veloxis Pharmaceuticals, Inc., through University of Arizona to conduct research and analysis for this study. Tyler is employed by Veloxis Pharmaceuticals, Inc.

Some of the data reported and used in this research were available from the US Renal Data System, the US Bureau of Labor Statistics, and the Agency for Healthcare Research and Quality's Healthcare Cost and Utility Project. The interpretation and reporting of these data are the responsibility of the authors and in no way should be seen as an official policy or interpretation of the US government.

\section{ACKNOWLEDGMENTS}

The authors thank and acknowledge University of Arizona students Brooke Tavares and Zufan Yegezu for their help to identify hospitalization costs associated with serious adverse events.

\section{REFERENCES}

1. Kasiske BL, Zeier MG, Chapman JR, et al. KDIGO clinical practice guideline for the care of kidney transplant recipients: a summary. Kidney Int. 2010;77(4):299-311. doi:10.1038/ki.2009.377

2. Chen L, Prasad GVR. CYP3A5 polymorphisms in renal transplant recipients: influence on tacrolimus treatment. Pharmacogenomics Pers Med. 2018;11:2333. doi:10.2147/PGPM.S107710

3. van Gelder T, Meziyerh S, Swen JJ, de Vries APJ, Moes DJAR. The clinical impact of the $\mathrm{C}(0) / \mathrm{D}$ ratio and the CYP3A5 genotype on outcome in tacrolimus treated kidney transplant recipients. Front Pharmacol. 2020;11:1142. doi:10.3389/fphar.2020.01142
4. Brunet M, van Gelder T, Åsberg A, et al. Therapeutic drug monitoring of tacrolimus-personalized therapy: second consensus report. Ther Drug Monit. 2019;41(3):261-307. doi:10.1097/ FTD.0000000000000640

5. Khan AR, Raza A, Firasat S, Abid A. CYP3A5 gene polymorphisms and their impact on dosage and trough concentration of tacrolimus among kidney transplant patients: a systematic review and meta-analysis. Pharmacogenomics J. 2020;20(4):553-62. doi:10.1038/ s41397-019-0144-7

6. Wang P, Mao Y, Razo J, et al. Using genetic and clinical factors to predict tacrolimus dose in renal transplant recipients. Pharmacogenomics. 2010;11(10):1389-402. doi:10.2217/pgs.10.105

7. Jacobson PA, Oetting WS, Brearley AM, et al. Novel polymorphisms associated with tacrolimus trough concentrations: results from a multicenter kidney transplant consortium. Transplantation. 2011;91(3):300-08. doi:10.1097/ TP.0b013e318200e991

8. Trofe-Clark J, Brennan DC, West-Thielke P, et al. Results of ASERTAA, a randomized prospective crossover pharmacogenetic study of immediate-release versus extended-release tacrolimus in African American kidney transplant recipients. Am J Kidney Dis. 2018;71(3):315-26. doi:10.1053/j.ajkd.2017.07.018

9. Bechstein WO. Neurotoxicity of calcineurin inhibitors: impact and clinical management. Transpl Int. 2000;13(5):31326. doi:10.1007/s001470050708

10. Cherala G, Munar MY, Naher A, Al-Uzri A. Tacrolimus pharmacokinetics in Hispanic children after kidney transplantation. Transplant Proc. 2011;43(10):3708-12. doi:10.1016/j. transproceed.2011.09.016

11. Claudio-Campos K, Duconge J, Cadilla CL, Ruaño G. Pharmacogenetics of drug-metabolizing enzymes in US Hispanics. Drug Metab Pers Ther. 2015;30(2):87-105. doi:10.1515/ dmdi-2014-0023
12. Kolb M, Offer K, Jin Z, et al. Risk factors for subtherapeutic tacrolimus levels after conversion from continuous intravenous infusion to oral in children after allogeneic hematopoietic cell transplantation. Biol Blood Marrow Transplant. 2016;22(5):957-61. doi:10.1016/j. bbmt.2016.02.005

13. Holm P, Buur A, Elema MO, Mollgaard B, Holm JE, Schultz K. MeltDose1 technology. United States Patent. May 15, 2007. Accessed August 10, 2020. https://patft.uspto.gov/ netacgi/nph-Parser?Sect1 $=$ PTO2\&Se ct2=HITOFF\& $\mathrm{p}=1 \& \mathrm{u}=\% 2$ Fnetahtml\%2 FPTO\%2Fsearch-bool.html\& $\mathrm{r}=1 \& \mathrm{f}=\mathrm{G}$ $\& \mathrm{l}=50 \& \mathrm{co1}=\mathrm{AND} \& \mathrm{~d}=$ PTXT\&s1=7217431. $\underline{\mathrm{PN} . \& O S=\mathrm{PN} / 7217431 \& \mathrm{RS}=\mathrm{PN} / 7217431}$

14. Nigro V, Glicklich A, Weinberg J. Improved bioavailability of MELTDOSE once-daily formulation of tacrolimus (LCP-Tacro) with controlled agglomeration allows for consistent absorption over 24 hrs: a scintigraphic and pharmacokinetic evaluation (Abstract \#B1034). Am J Transplant. 2013;13(S5):339. doi:10.1111/ ajt.12266

15. Gaber AO, Alloway RR, Bodziak K, Kaplan B, Bunnapradist S. Conversion from twice-daily tacrolimus capsules to once-daily extended-release tacrolimus (LCPT): a phase 2 trial of stable renal transplant recipients. Transplantation. 2013;96(2):191-97. doi:10.1097/ TP.0b013e3182962cc1

16. Alloway RR, Mulgaonkar S, Ueda K, Cohen D, Kaplan B. A phase 2 randomized study of the pharmacokinetics, safety and efficacy of LCP-Tacro tablets oncea-day vs Prograf capsules twice-a- day in de novo kidney transplants (Abstract \#1106). Am J Transplant. 2011;11(S2):355. doi:10.1111/j.1600-6143.2011.03534.x

17. Budde K, Bunnapradist S, Grinyo JM, et al. Novel once-daily extended-release tacrolimus (LCPT) versus twice-daily tacrolimus in de novo kidney transplants: one-year results of Phase III, doubleblind, randomized trial. Am J Transplant. 2014;14(12):2796-806. doi:10.1111/ajt.12955 
18. Bunnapradist S, Ciechanowski K, West-Thielke $\mathrm{P}$, et al. Conversion from twice-daily tacrolimus to once-daily extended release tacrolimus (LCPT): the phase III randomized MELT trial. Am J Transplant. 2013;13(3):760-69. doi:10.1111/ ajt.12035

19. Morrissey PE, Reinert S, Yango A, Gautam A, Monaco A, Gohh R. Factors contributing to acute rejection in renal transplantation: the role of noncompliance. Transplant Proc. 2005;37(5):2044-47. doi:10.1016/j.transproceed.2005.03.017

20. Belaiche S, Décaudin B, Dharancy S, Noel C, Odou P, Hazzan M. Factors relevant to medication non-adherence in kidney transplant: a systematic review. Int J Clin Pharm. 2017;39(3):582-93. doi:10.1007/s11096-017-0436-4

21. Rebafka A. Medication adherence after renal transplantation-a review of the literature. J Ren Care. 2016;42(4):239-56. doi:10.1111/jorc.12181

22. Bunnapradist S, Guerra G, Patel S, et al. Safety and efficacy of conversion from immediate-release tacrolimus (IR-TAC) to LCP-tacrolimus (LCPT) in Hispanic kidney transplant patients (\#FRPO1188). Presented at: American Society of Nephrology Kidney Week; November 2019; Washington DC.

23. Villacana R, Guerra G, Moten M, et al. Safety and efficacy of LCPtacrolimus (LCPT) in Hispanic de novo kidney transplant recipients (KTR) (\#FRPO1186). Presented at: American Society of Nephrology Kidney Week; November 2019; Washington DC.

24. Bunnapradist S, Rostaing L, Alloway RR, et al. LCPT once-daily extended-release tacrolimus tablets versus twice-daily capsules: a pooled analysis of two phase 3 trials in important de novo and stable kidney transplant recipient subgroups. Transpl Int. 2016;29(5):603-11. doi:10.1111/tri.12770
25. Rostaing L, Bunnapradist S, Grinyó JM, et al. Novel once-daily extended-release tacrolimus versus twice-daily tacrolimus in de novo kidney transplant recipients: two-year results of phase 3 , doubleblind, randomized trial. Am J Kidney Dis. 2016;67(4):648-59. doi:10.1053/j. ajkd.2015.10.024

26. Beimler J, Zeier M. Borderline rejection after renal transplantation-to treat or not to treat. Clin Transplant. 2009;23(Suppl 21):19-25. doi:10.1111/j.1399-0012.2009.01105.x

27. Brennan D, Malon A. Kidney transplantation in adults: treatment of acute T cell-mediated (cellular) rejection of the renal allograft. In Lam AQ, ed. UpToDate. Updated February 2020. Accessed March 24, 2020. https:// www.uptodate.com/contents/ kidney-transplantation-in-adults-treatment-of-acute-t-cell-mediated-cellularrejection-of-the-renal-allograft

28. Kim S. The effect of steroid pulse therapy for the reduction of acute rejection episode in subclinical borderline changes: an open-label, randomized clinical trial. ClinicalTrials.gov. Accessed March 24, 2020. https://clinicaltrials.gov/ ct2/show/NCT02664493

29. Leblanc J, Subrt P, Paré M, et al. Practice patterns in the treatment and monitoring of acute $t$ cell-mediated kidney graft rejection in Canada. Can J Kidney Health Dis. 2018;5:2054358117753616. doi:10.1177/2054358117753616

30. Nankivell BJ, Agrawal N, Sharma A, et al. The clinical and pathological significance of borderline T cellmediated rejection. Am J Transplant. 2019;19(5):1452-63. doi:10.1111/ajt.15197

31. Reschen ME, Mazzella A, Sharples E. A retrospective analysis of the utility and safety of kidney transplant biopsies by nephrology trainees and consultants. Ann Med Surg 2012. 2018;28:6-10. doi:10.1016/j. amsu.2018.02.001
32. Roufosse C, Simmonds N, Clahsen-van Groningen M, et al. A 2018 reference guide to the Banff Classification of Renal Allograft Pathology. Transplantation. 2018;102(11):1795-814. doi:10.1097/TP.0000000000002366

33. Whittier WL, Gashti C, Saltzberg S, Korbet S. Comparison of native and transplant kidney biopsies: diagnostic yield and complications. Clin Kidney J. 2018;11(5):616-22. doi:10.1093/ckj/sfy051

34. Whittier W, Korbet S. The kidney biopsy. In: Lam AQ, ed. UpToDate. May 2019. Updated December 15, 2020. Accessed June 9, 2021. https://www.uptodate.com/contents/the-kidney-biopsy

35. Agency for Healthcare Research and Quality. National Inpatient Sample (NIS). Healthcare Cost and Utilization Project (HCUP). 2016. Accessed March 24, 2020. http://hcupnet.ahrq.gov/

36. Investigational New Drug Application. 21 e-C.F.R. §312.32. April 2020. Accessed June 9, 2021. https://ecfr.federalregister.gov/current/title-21/chapter-I/ subchapter-D/part-312

37. IBM Watson Health. IBM Micromedex RED BOOK database. Accessed February 20, 2020. https://www.ibm.com/watson/health/provider-client-training/ micromedex-red-book/

38. Symphony Health. PatientSource ${ }^{\circledR}$ sales sheet. November 2019.

Accessed June 9, 2021. https://symphonyhealth.prahs.com/insights/ patientsource-sales-sheet

39. Centers for Medicare \& Medicaid Services. Healthcare Common Procedural Coding System (HCPCS) database. Accessed March 24, 2020. https:// www.cms.gov/Medicare/Coding/ MedHCPCSGenInfo

40. Centers for Medicare \& Medicaid Services. Physician fee schedule. 2020. Accessed March 24, 2020. https://www.cms.gov/Medicare/ Medicare-Fee-for-Service-Payment/ PhysicianFeeSched 
41. US Renal Data System 2019 annual data report: epidemiology of kidney disease in the United States. Am J Kidney Dis. Published online October 31, 2019. doi:10.1053/j.ajkd.2019.09.002

42. US Bureau of Labor Statistics. Consumer Price Index data tables. 20162019. Accessed March 24, 2020. https:// www.bls.gov/cpi/

43. Wu O, Levy AR, Briggs A, Lewis G, Jardine A. Acute rejection and chronic nephropathy: a systematic review of the literature. Transplantation. 2009;87(9):1330-39. doi:10.1097/

TP.0b013e3181a236e0
44. Axelrod DA, Schnitzler MA, Xiao H, et al. The changing financial landscape of renal transplant practice: a national cohort analysis. Am J Transplant. 2017;17(2):377-89. doi:10.1111/ajt.14018

45. American Society of Transplantation. TxPharm and PCOP issue statement on tacrolimus shortage. July 24, 2019. Accessed August 8, 2020. https://www. myast.org/txpharm-and-pcop-issuestatement-tacrolimus-shortage
46. US Food and Drug Administration. FDA drug shortages. Accessed August 8, 2020. https://www.accessdata. fda.gov/scripts/drugshortages/ dsp_ActiveIngredientDetails.

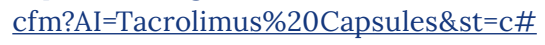

